\title{
Membranous CD44v6 is upregulated as an early event in colorectal cancer: Downregulation is associated with circulating tumor cells and poor prognosis
}

\author{
AGAPI KATAKI ${ }^{1}$, VASSILIS G. GIANNAKOULIS ${ }^{1}$, ANASTASIA DERVENTZI ${ }^{1}$, \\ KONSTANTINOS PAPIRIS ${ }^{2}$, EYTHIMIOS KONIARIS ${ }^{3}$ and MANOUSOS KONSTADOULAKIS ${ }^{4}$ \\ ${ }^{1}$ First Department of Propaedeutic Surgery; Departments of ${ }^{2}$ Endoscopy and ${ }^{3}$ Pathology, \\ Hippokration General Hospital of Athens, Athens 11527; ${ }^{4}$ Second Surgery Clinic, Aretaieio Hospital, \\ Athens Medical School, National and Kapodistrian University of Athens, Athens 11528, Greece
}

Received November 14, 2020; Accepted July 1, 2021

DOI: 10.3892/ol.2021.13081

\begin{abstract}
Previous studies have reported that CD44 variant $6(\mathrm{CD} 44 \mathrm{v} 6)$ and metastasis-associated protein 1 (MTA1) are contributing factors to cancer progression. The present study aimed to evaluate the expression profiles for associations with patients' demographic data, clinicopathological characteristics, the presence of partial epithelial-to-mesenchymal transition (pEMT), metastatic potential based on the presence of $\mathrm{CK}_{20} 0^{+} \mathrm{CEA}^{+} \mathrm{CXCR} 4^{+}$circulating tumor cells (CTCs) and prognosis (median follow-up, 45 months). Thus, frozen tissue samples from 31 patients with stage I-III colorectal cancer (CRC), 15 benign colorectal polyps and seven normal colorectal tissues were analyzed to detect membranous (m)CD44v6 and MTA1 expression via flow cytometry. The results demonstrated that the mCD44v6 and MTA1 expression profiles were significantly correlated $\left(r_{s}=+0.786, P<0.001\right)$. Notably, MTA1 expression was not associated with any of the clinicopathological characteristics assessed. The percentage of mCD44v6-positive cells within tumors was higher in the right-sided cancer lesions $(\mathrm{P}=0.014)$, suggesting that proximal and distal CRCs are distinct clinicopathological entities. Furthermore, downregulated mCD44v6 expression was significantly associated with the presence of CTCs $(\mathrm{P}=0.017)$. This association was stronger for pEMT (co-expression of $\mathrm{N}$ - and $\mathrm{E}$-cadherin mRNAs) primary lesions $(\mathrm{P}=0.009)$. In addition, patients with CRC with low levels of mCD44v6 had unfavorable survival outcomes $(\mathrm{P}=0.037)$. Taken together, these results suggest that targeted analysis of membranous
\end{abstract}

Correspondence to: Dr Agapi Kataki, First Department of Propaedeutic Surgery, Hippokration General Hospital of Athens, Vasilissis Sofias 114, Athens 11527, Greece

E-mail: akataki@med.uoa.gr

Key words: membranous CD44 variant 6, metastasis-associated protein 1, circulating tumor cells, polyps, colorectal cancer, tumor microenvironment
CD44v6 as opposed to membranous-cytoplasmic expression is important in determining the prognosis of patients with CRC. Furthermore, downregulated $\mathrm{mCD} 44 \mathrm{v} 6$ expression in malignancies presenting CTCs reinforces the importance of tumor-stroma reciprocal influence during the metastatic process and encourages the assessment of relevant therapeutic strategies.

\section{Introduction}

Colorectal cancer (CRC) is the third leading cause of cancer deaths worldwide, as patients with lymph node invasion or metastatic lesions have poor prognosis despite the remarkable progress in therapeutic advances (1). Therefore in search for the development of effective molecular targeted therapies, it is pivotal to identify protein patterns that play a key role in CRC progression and in the interaction between cancer cells and the surrounding stroma which is considered essential for tumor survival and advancement.

A crucial concept in colorectal carcinogenesis is the adenoma-carcinoma sequence, which describes the gradual step-wise transformation of normal mucosa to cancer over time and concludes with metastasis (2). The metastatic process seems to be facilitated through the epithelial-mesenchymal transition (EMT) of cancer cells during which, cells lose their cell to cell adhesion capacity (3). Through this process, cells convert to an anaplastic form characterized by the upregulation of $\mathrm{N}$-cadherin and the downregulation of E-cadherin (the so-called "cadherin switch') but as EMT seems to represent a spectrum, cells often acquire a 'hybrid' EMT phenotype (partial EMT, pEMT) $(4,5)$. Importantly, pEMT cells are considered to harbor the best characteristics in terms of metastatic potential $(6,7)$.

CD44 variant 6 (CD44v6), and metastasis-associated protein 1 (MTA1) have been considered as possible inducers of the EMT process $(8,9)$. CD44v6 constitutes a multifunctional transmembrane protein involved in a variety of biological processes, including cell growth, apoptosis, migration and angiogenesis (10). Notably, its extracellular domain allows for extensive interaction with the tumor microenvironment, with hyaluronic acid being its major ligand (11). Several 
studies have shown that cancer epithelial cells favor the presence of a hyaluronic acid rich tissue structure in their stroma and that following binding of hyaluronic acid to mCD44v6, several pathways promoting growth and cell-survival of neoplastic cells are activated $(12,13)$. Therefore, in our study, we focused on membranous (rather than intracellular) CD44v6 (mCD44v6) expression. In contrast, MTA1 is considered to exert its functions intracellularly and mainly in the nucleus, where it has been reported to act as both a transcriptional corepressor and coactivator (14).

Circulating tumor cells (CTCs) are considered an intermediate step between the primary and metastatic cancer lesion and their role in cancer progression has been a major area of interest $(15,16)$. Previous studies have utilized cytokeratin-20 (CK20) and carcinoembryonic antigen (CEA) expression to detect CTCs in CRC $(17,18)$. Furthermore, the expression of C-X-C chemokine receptor type 4 (CXCR4) was shown to allow CTCs to follow stromal cell-derived factor 1 (SDF1) gradients and seed tumors at remote sites (19). As liver tissue is a common CRC metastatic site, which constitutively expresses SDF1, the expression of CXCR4 by CTCs was also considered in our study $(20,21)$.

Within this context, the present study analyzed the expression profiles of mCD44v6 and MTA1 proteins in normal colorectal tissues, benign polyps, and up to stage III CRC tissues, using flow cytometry. Their expression profiles were evaluated in association with clinicopathological variables, pEMT manifestation, proliferation, as well as the presence of CTCs. Finally, their prognostic role in disease progression was assessed.

\section{Materials and methods}

Patients and tissues. The tissue specimens analyzed in the present study consisted of: a) surgically resected cancer tissue, as determined by a pathologist, from $31 \mathrm{CRC}$ patients as well as bioptic material from b) 15 colorectal polyps and c) 7 normal colorectal tissues, excised during endoscopic colonoscopy performed for diagnostic purposes. The study was approved by the Hippocration Hospital scientific committee (18601/2019). The cohort of CRC patients enrolled was from a previous study, in which apart from the tissue, a peripheral blood sample was acquired $24 \mathrm{~h}$ before laparotomy in order to detect CTCs (18). From the initial cohort, only those patients with available biologic material, as well as accessible relevant data, were included herein. The assay used in that study had a sensitivity of 5 cells per $\mathrm{ml}$ peripheral blood as derived from the spiking experiments with HT29 cells. In the newly recruited patients subjected to endoscopic colonoscopy the significance of the study and its safety were explained, and their informed consent was obtained as well. Demographic and clinical data were collected from the patients' medical files and are presented in Table I. Staging was performed according to 8th edition of the American Joint Committee on Cancer staging system manual. All patients received the standard surgical and medical care available at the time of diagnosis. CRC patients were followed-up for a median period of 45 months (14-54) during which, 6 deaths and 5 metastases occurred, with a composite event of death (all-cause) or metastasis occurring 8 times.
Flow cytometry analysis for CD44v6 and MTA1. Tissue samples wetted with $1 \mathrm{ml} 1 \mathrm{X}$ PBS were mechanically disaggregated by placing them into a Medicon (BD Biosciences) and inserted in the Medimachine (BD Biosciences) for $45 \mathrm{sec}$ at $100 \mathrm{rpm}$. Cell suspensions were filtered using a Filcon (BD Biosciences), washed twice with $1 \mathrm{X}$ PBS and divided at a concentration of approximately $1 \times 10^{7}$ cells $/ \mathrm{ml}$ into aliquots for staining. For detection of mCD44v6 positive cells, cell aliquots were incubated with CD44v6 (Bio-Rad MCA1730, dilution 1:500) for $1 \mathrm{~h}$ at room temperature (RT) in an orbital shaker at low speed. Cells were then washed and the percentage of cells expressing mCD44v6 was detected following a $60 \mathrm{~min}$ incubation with a goat anti-mouse IgG1: FITC secondary antibody (Bio-Rad STAR132F; dilution 1:100).

For intracellular labelling, cells fixation-permeabilization was performed using $1 \%$ paraformaldeyde- $1 \%$ saponin in $1 \mathrm{X}$ PBS for $20 \mathrm{~min}$ at $4^{\circ} \mathrm{C}$ before cells were incubated with MTA1 antibody (SantaCruz Biotechnology SC-17773 dilution 1:500) and stained with a Polyclonal Goat anti-mouse-RPE Goat F(ab)2 secondary antibody (Dako R0480, dilution 1:100). Unstained cells were processed in parallel and included as references during analysis as well as cells stained only with the secondary antibodies. Flow cytometry analysis was performed using a Coulter Epics XL-MCL Flow Cytometer and its integrated SYSTEM II ${ }^{\mathrm{TM}}$ Software.

Immunohistochemical detection of mCD44v6 and MTA1. Five- $\mu \mathrm{m}$ sections of the paraffin-embedded tissue blocks from patients of the same cohort were used. Sections mounted on superfrost glass slides, were deparaffinized in xylol, rehydrated in a graded ethanol series, and then subjected to microwave antigen retrieval [2x8 $\mathrm{min}, 800 \mathrm{w}$, in Tris-EDTA, pH $9.0(10 / 1 \mathrm{mM})]$. Endogenous peroxidase activity was blocked using $0.3 \%$ hydrogen peroxide. Sections were incubated at $4^{\circ} \mathrm{C}$ overnight with the following primary antibodies at the specified dilutions mCD44v6 (Bio-Rad MCA1730, dilution 1:100), MTA1 (Santa Cruz Biotechnology SC-17773 dilution 1:200). The detection was performed according to the ChemMateEnVision system (DAKO EnVision Detection Systems, Peroxidase/DAB, Rabbit/Mouse) protocol. Sections were then counterstained with Mayer hematoxylin, dehydrated, cleared mounted using toluene-free mounting medium (Dako CS705) and observed by means of a Zeiss Axiostar Microscope.

Percentage of cells in the $S$ phase of cell cycle. Coulter DNA Prep Kit (Beckman Coulter) was used for quantitative measurements of DNA content in malignant tissues. Fluorescence-stained samples were analyzed by flow cytometry with a Coulter Epics XL-MCL flow cytometer and data were evaluated with MultiCycle AV Software (Phoenix Flow Systems). A minimum of $1 \times 10^{4}$ events were analyzed for each sample. DNA determinations provided information on the percentage of cells in the $S$ phase of the cycle, which was subsequently used as a marker of cellular proliferation among malignant tissues.

$N$ - and $E$-cadherin $m R N A$ detection in primary tissue samples. Total RNA was extracted from cancer lesions using the RNeasy Mini Kit (Qiagen), reverse transcribed using SuperScript II 
Table I. Patient characteristics.

\begin{tabular}{|c|c|}
\hline Characteristic & Value \\
\hline \multicolumn{2}{|l|}{ Number of females, $n$} \\
\hline Cancer group & 16 \\
\hline Polyps group & 6 \\
\hline Normal group & 1 \\
\hline \multicolumn{2}{|l|}{ Mean age, years (SD) } \\
\hline Cancer group & $\begin{array}{c}70.48(7.83) ; \\
\text { Min, 53; Max, } 87\end{array}$ \\
\hline Polyps group & $\begin{array}{c}63.40(9.55) ; \\
\text { Min, } 49 ; \operatorname{Max}, 80\end{array}$ \\
\hline Normal group & $\begin{array}{c}60.43(17.90) ; \\
\text { Min, 24; Max, } 78\end{array}$ \\
\hline \multicolumn{2}{|l|}{ TNM staging } \\
\hline 0 & 3 \\
\hline $\mathrm{I}$ & 7 \\
\hline II & 11 \\
\hline III & 10 \\
\hline Lymph node metastasis & 10 \\
\hline \multicolumn{2}{|l|}{ Grade } \\
\hline 1 & 5 \\
\hline 2 & 23 \\
\hline 3 & 3 \\
\hline Median cancer tumor diameter, $\mathrm{cm}$ & $\begin{array}{c}4.5 \\
(\operatorname{Min}, 0.6 ; \operatorname{Max}, 16)\end{array}$ \\
\hline Right-sided location & 9 \\
\hline Median follow-up, months & 45 \\
\hline
\end{tabular}

(Min, 14; Max, 54)

TNM, tumor-node-metastasis; Cancer, 31 cases; polyps, 15 cases; normal, 7 cases.

RNAse Reverse Trascriptase (Invitrogen Life Technologies) as described in Lagoudianakis et al (18) and subjected to sequential PCR amplifications with primers designed specific for N- and E-cadherin mRNA (Table SI). cDNA quality was assessed by conventional PCR for the ubiquitous housekeeping gene $\beta 2$-microglobulin and the same amount was subjected to conventional RT-PCR. Primers and annealing temperatures are presented in supplementary material (Table SI). RT-PCR products were analyzed and scored positive/negative on ethidium bromide stained $2 \%$ agarose gel.

CXCR4 detection in peripheral circulating cells. For the detection of CXCR4 mRNA in the tumor cells present in the systemic circulation conventional RT-PCR was used as previously described for CEA and CK20 expression in Lagoudianakis et al (18). Primers and amplification conditions are also included in Table SI.

Statistical analysis. The mean with standard deviation, median and minimum-maximum values, as well as graphs (box plots, scatter plots, Kaplan Meier curve). flow cytometric plots and immunohistochemistry images are used for data presentation and visualization. Statistical analysis was performed using SPSS 25.0 (SPSS, Inc.). Flow cytometric data were treated as quantitative data and assessed using non-parametric methods (Mann-Whitney U test, Spearman's rank correlation coefficient). The chi-squared or Fisher's exact test was used for qualitative data analysis. Kruskal-Wallis test, with post hoc pairwise comparisons using the Dunn-Bonferroni method were performed to compare protein expression among normal tissues, benign polyps and colorectal adenocarcinomas. Cox proportional hazards regression was used to identify predictors for a composite endpoint comprising of metastasis or all-cause mortality. A simple and a multivariable model were utilized, with the multivariable model including predictors with $\mathrm{P}<0.1$ in the simple model. Kaplan-Meier curve, with long-rank test for differences in survival functions between groups was applied for metastasis-free survival. A P-value $\leq 0.05$ was considered to indicate a statistically significant difference.

\section{Results}

$m C D 44 v 6$ and MTA1 expression from normal to malignant colorectal tissues. The percentages of mCD44v6 and MTA1 positive cells were analysed using the non-parametric tests to compare the expression profile of each protein in colonic normal epithelium, polyp and cancer epithelium. Although no statistically significant differences occurred in normal tissues when compared to benign polyps, analysis of normal and polyp tissues compared to colorectal adenocarcinoma ones revealed a statistically significantly difference for both protein expression profiles ( $\mathrm{P}<0.001$; Figs. 1A-D and 2A-D). Immunohistochemical detection of corresponding paraffin sections further supported the results of the flow cytometry analysis (Figs. 1E-G and 2E-G). In the present cohort the expression profiles of these two proteins significantly correlated $\left(r_{\mathrm{s}}=+0.786, \mathrm{P}<0.001\right.$; Fig. 3).

Specifically, in CRC patients, $\mathrm{mCD} 44 \mathrm{v} 6$ protein expression was significantly higher as compared to patients with benign colorectal polyps $[24.61 \%(10.12)$ vs. $8.61 \%$ (6.72), median: 25 vs. $8.00 \%, \mathrm{P}<0.001]$ and normal tissues [24.61\% (10.12) vs. $6.82 \%$ (1.51), median: 25 vs. $6.81 \%, \mathrm{P}<0.001]$. Similarly, CRC patients expressed higher MTA1 in comparison to the group of benign polyps [49.35\% (14.86) vs. $16.50 \%$ (11.71), median: 50.00 vs. $10.00 \%, \mathrm{P}<0.001]$ and normal tissues $[49.35 \%$ (14.86) vs. $2.29 \%$ (0.51), median: 50 vs. $2.4 \%, \mathrm{P}<0.001]$.

No significant associations were observed between mCD44v6 or MTA1 protein expression and TNM stage, grade or nodal status. However, mCD44v6 expression was increased in those patients presenting the primary lesion in the right colon [31.56\% (9.51) vs. $21.77 \%$ (9.10), median: 30 vs. $22 \%$; $\mathrm{P}=0.014) ;$ Fig. 4].

Primary tumor site biomarker expression and CTCs. Patients with CRC were divided in two subgroups according to the presence of circulating cells expressing CK20 and CEA mRNA $\left(\mathrm{CK} 20^{+} \mathrm{CEA}^{+}\right)$in their preoperative blood samples. Twenty-four were positive for CK20 (77.4\%) and thirteen for CEA $(41.9 \%)$ mRNA expression whereas, twelve blood samples were double positive $\left(\mathrm{CK}^{2} 0^{+} \mathrm{CEA}^{+}: 38.7 \%\right)$.

The percentage of cells expressing mCD44v6 protein in the primary lesion was significantly lower in patients with detectable circulating $\mathrm{CK} 20^{+} \mathrm{CEA}^{+}$cells compared to those 

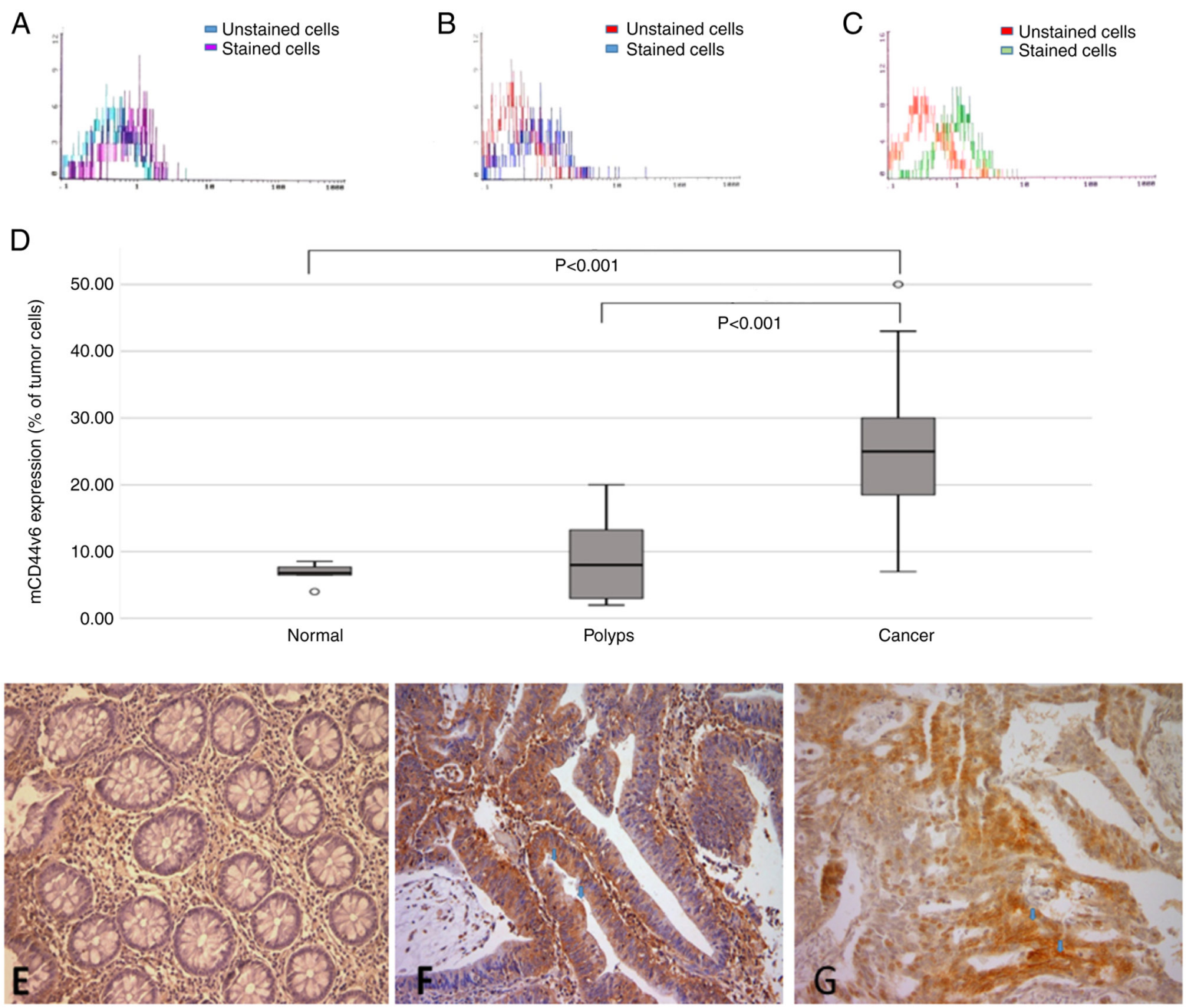

Figure 1. mCD44v6 expression analysis by flow cytometry and immunohistochemistry. Flow cytometric analysis of mCD44v6 expression in (A) normal epithelium, (B) benign polyps and (C) malignant colorectal lesions. (D) Statistical analysis revealed that the percentage of mCD44v6-positive cells was signifficantly induced in colorectal cancer compared with benign polyps and normal epithelium. (E) Immunohistochemistry analysis of mCD44v6 expression in normal colonic mucosa exhibited neither cytoplasmic or membranous expression. (F) Immunohistochemistry analysis of mCD44v6 expression in colonic polyp exhibited faint cytoplasmic stain and focal luminal membranous expression (blue arrows). (G) Immunohistochemistry analysis of mCD44v6 expression in colorectal cancer tissues exhibited an intense membranous stain in both luminal and basolateral sides of neoplastic cells (blue arrows). Magnifcation, x200. mCD44v6, membranous CD44 variant 6 .

with no detectable CTCs $[19.83 \%$ (8.41) vs. 27.63\% (10.13),

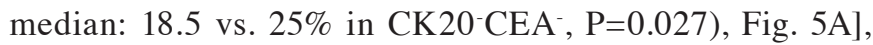
but remained significantly higher as compared to the group of benign colorectal polyps [19.83\% (8.41) vs. $8.61 \%(6.72)$, median: 18.5 vs. $8 \%, \mathrm{P}=0.002]$. Similar results were found when patients with co-expression of CK20, CEA and CXCR4 in CTCs $(n=9 ; 29 \%)$ were analysed for the percentage of $\mathrm{mCD} 44 \mathrm{v} 6$ positive cells in their primary lesion [(18.44\% (8.82) vs. $27.14 \%$ (9.68), median: 18 vs. $25 \%, \mathrm{P}=0.017$; Fig. 5B)].

When the above-described subgroups of CRC patients were compared for the percentage of cells expressing MTA1 in the primary lesion no significant differences were detected $\left(\mathrm{CK} 20^{+} \mathrm{CEA}^{+}\right.$: $\mathrm{P}=0.49$; $\left.\mathrm{CK} 20^{+} \mathrm{CEA}^{+} \mathrm{CXCR} 4+: \mathrm{P}=0.5\right)$.

pEMT: Implication in the relationship of $m C D 44 v 6$ and CTCs. In order to examine whether the relationship of mCD44v6 and $\mathrm{CK} 20^{+} \mathrm{CEA}^{+} \mathrm{CXCR} 4^{+} \mathrm{CTC}$ is affected by the presence of pEMT, patients expressing both $\mathrm{E}$ - and $\mathrm{N}$-cadherin mRNAs in their primary malignant lesion were considered as manifesting pEMT $(n=19,61.3 \%)$. Within this group, patients with detectable CTCs in their blood stream presented lower percentage of cells expressing mCD44v6 [14.00\% (7.38) vs. $27.57 \%$ (8.72), median: 10 vs. $26 \% \mathrm{P}=0.009$; Fig. $6 \mathrm{~A}]$. A similar finding was not observed in the non-pEMT group [24.00\% (7.79) vs. $26.38 \%$ (11.80), median: 21.5 vs. $25 \%$, $\mathrm{P}=0.55$; Fig. 6B] indicating a role of $\mathrm{mCD} 44 \mathrm{v} 6$ expression in the way pEMT affects the metastatic potential of the tumor.

$m C D 44 v 6$ expression and event-free survival. In simple Cox-regression analysis of factors associated with event-free (metastasis ordeath) survival(Table II) only mCD44v6 expression was found significant $(\mathrm{HR}=0.915 ; 95 \%$, CI 0.843-0.994; $\mathrm{P}=0.035$ ). In multivariable regression analysis involving predictors with $\mathrm{P}<0.1$ in simple regression analysis (namely, tumor diameter and $\mathrm{mCD} 44 \mathrm{v} 6$ ), the significance of $\mathrm{mCD} 44 \mathrm{v} 6$ expression remained unchanged $(\mathrm{P}=0.017$; Table III).

The mean value of mCD44v6 expression in CRC patients with triple positive CTCs $\left(\mathrm{CK} 20^{+} \mathrm{CEA}^{+} \mathrm{CXCR} 4^{+}\right)$was used to characterize patients as positive $(\geq 18.44 \%$; $n=23)$ or negative $(<18.44 \%$; $n=8)$ for $\mathrm{mCD} 44 \mathrm{v} 6$ expression. Kaplan-Meier 

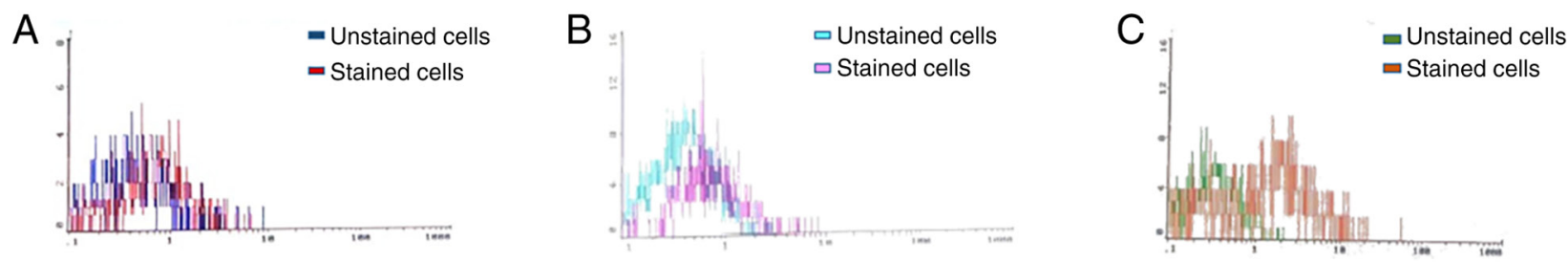

D
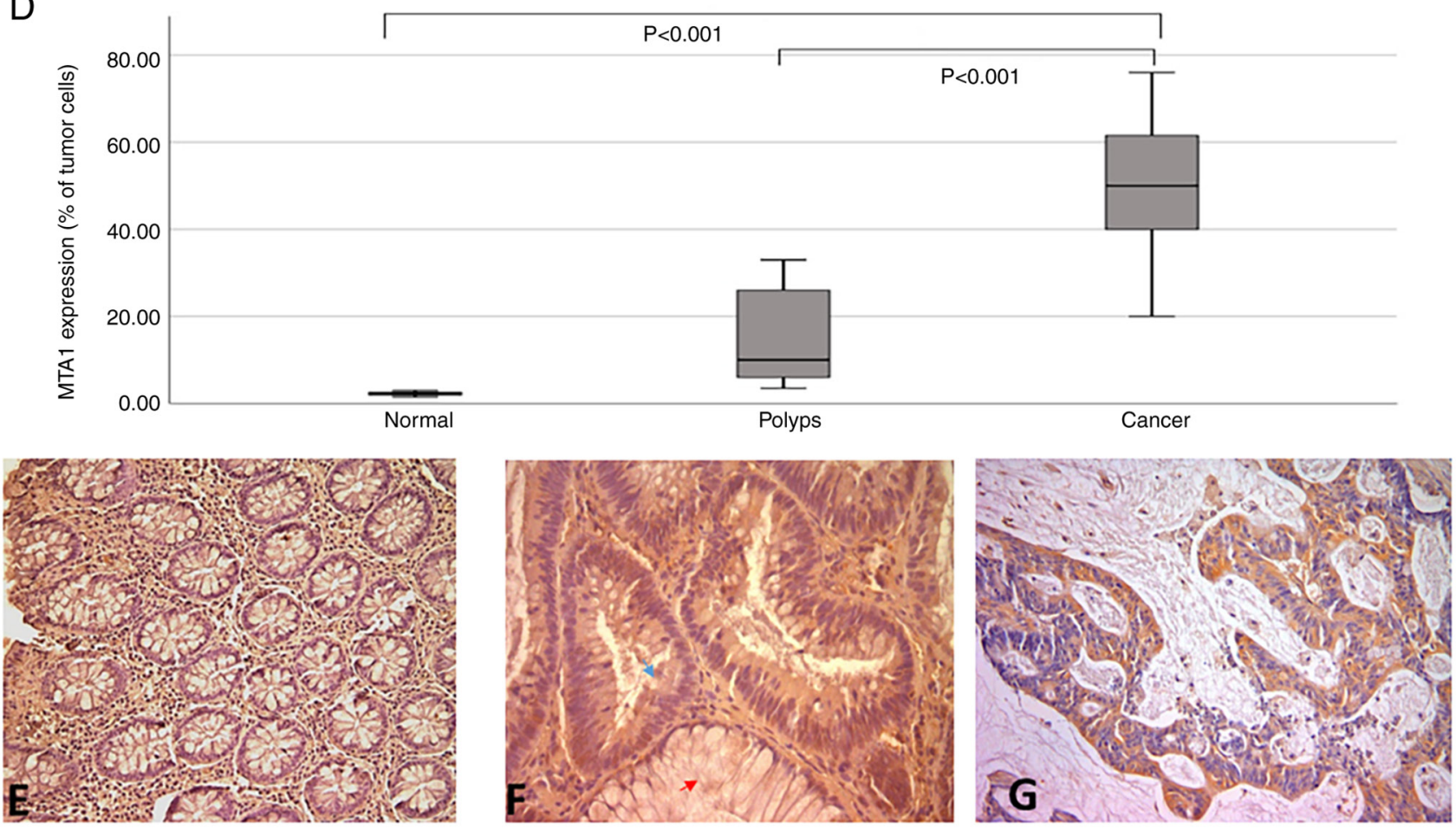

Figure 2. MTA1 expression analysis by flow cytometry and immunohistochemistry. Flow cytometric analysis of MTA1 expression in (A) normal epithelium, (B) benign polyps and (C) malignant colorectal lesions. (D) Statistical analysis revealed that the percentage of MTA1-positive cells was significantly induced in colorectal cancer compared with benign polyps and normal epithelium. (E) Immunohistochemistry analysis of MTA1 expression in normal colonic mucosa exhibited no cytoplasmic expression. (F) Immunohistochemistry analysis of MTA1 expression in colonic polyp exhibited weak cytoplasmic expression in adenomatous area (blue arrow) and no expression was observed in adjacent normal gland (red arrow). (G) Immunohistochemistry analysis of MTA1 expression in colorectal cancer tissues exhibited intense focal cytoplasmic stain. Magnification, x200. MTA1, metastasis-associated protein 1.

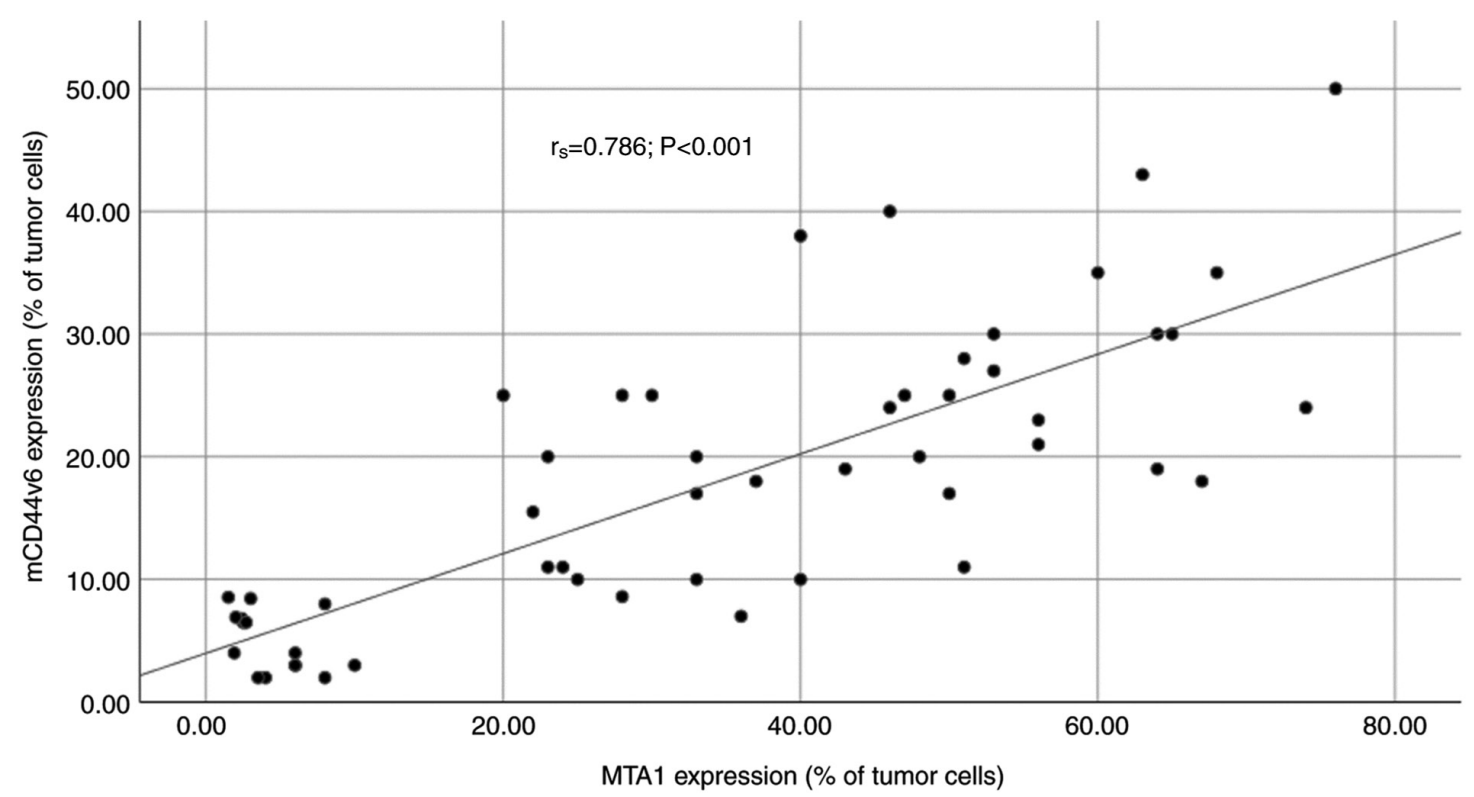

Figure 3. Spearman's correlation coefficient analysis. The expression profiles of $\mathrm{mCD} 44 \mathrm{v} 6$ and MTA1 proteins were signifcantly correlated $\left(\mathrm{r}_{\mathrm{s}}=0.786 ; \mathrm{P}<0.001\right)$. mCD44v6, membranous CD44 variant 6; MTA1, metastasis-associated protein 1. 


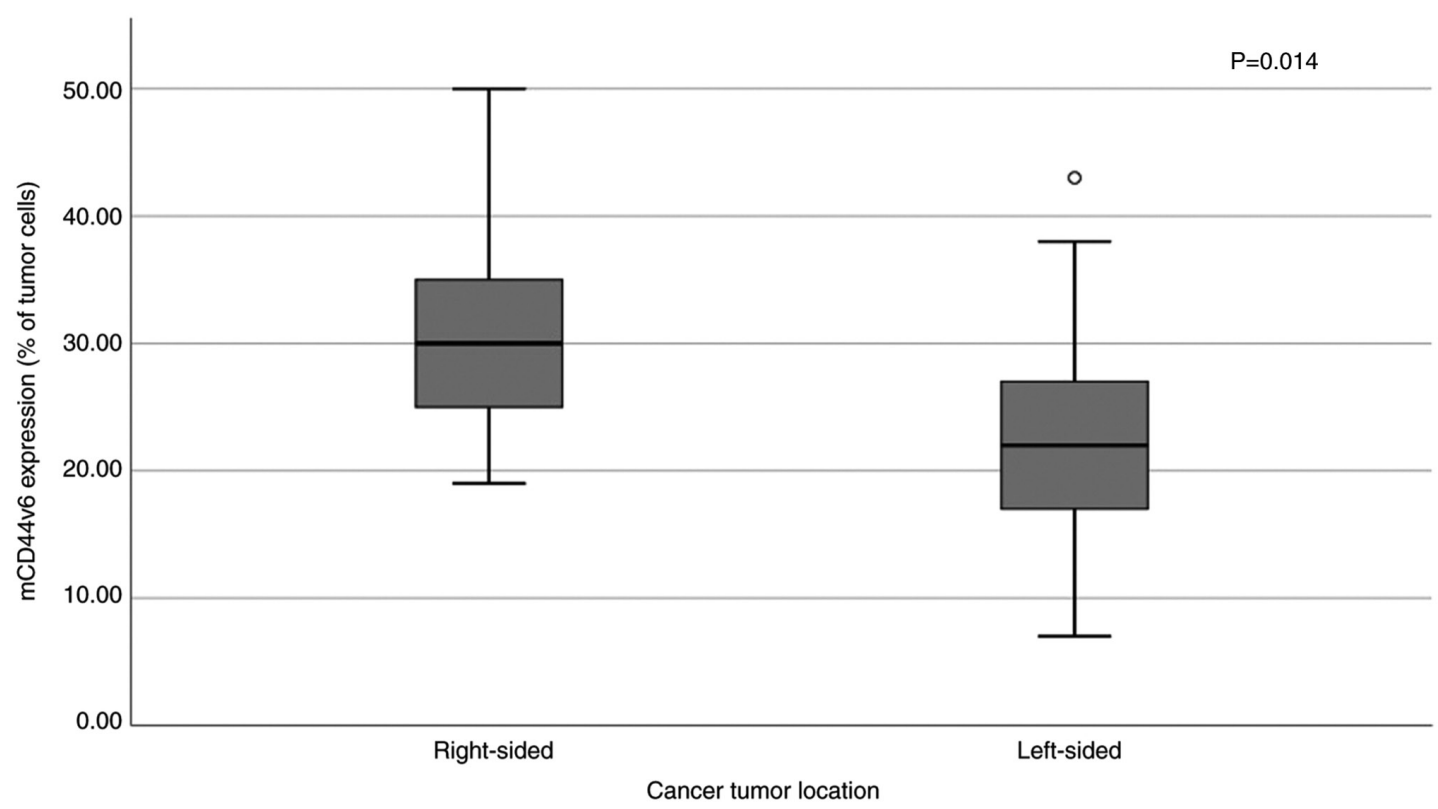

Figure 4. Differential expression of mCD44v6 between right- and left-sided colorectal cancer ( $\mathrm{P}=0.014)$. mCD44v6, membranous CD44 variant 6.
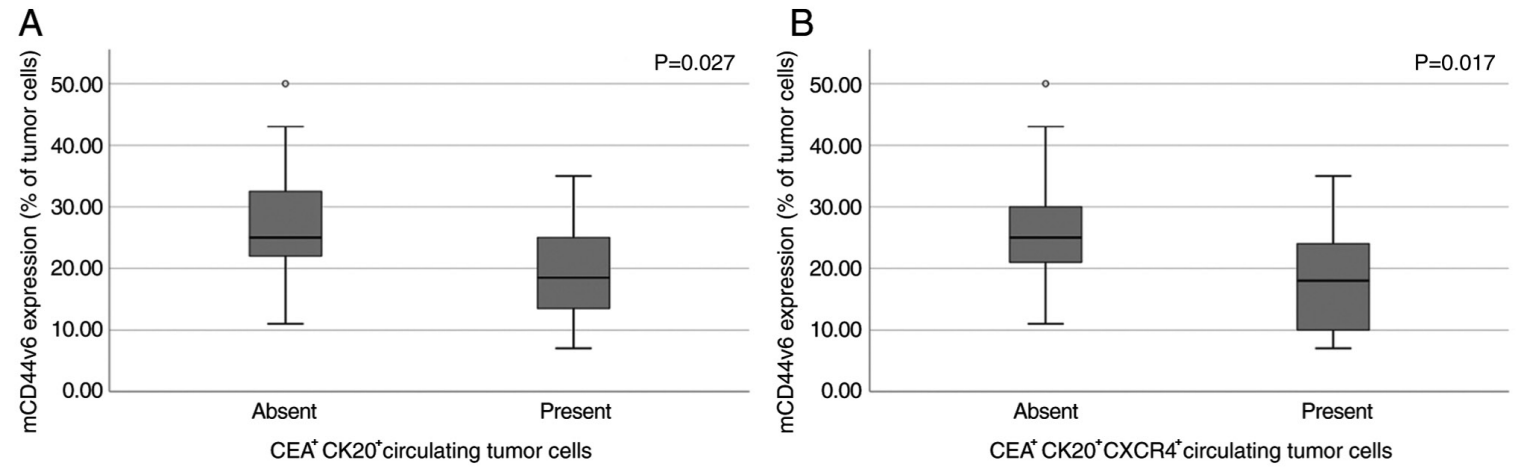

Figure 5. mCD44v6 expression in primary tumor site in relation to circulating tumor cells. Differential expression of mCD44v6 in the primary tumor site based on the presence or absence of (A) $\mathrm{CEA}^{+} \mathrm{CK} 20^{+}$and (B) $\mathrm{CEA}^{+} \mathrm{CK} 20^{+} \mathrm{CXCR} 4^{+}$circulating cells. mCD44v6, membranous CD44 variant 6.
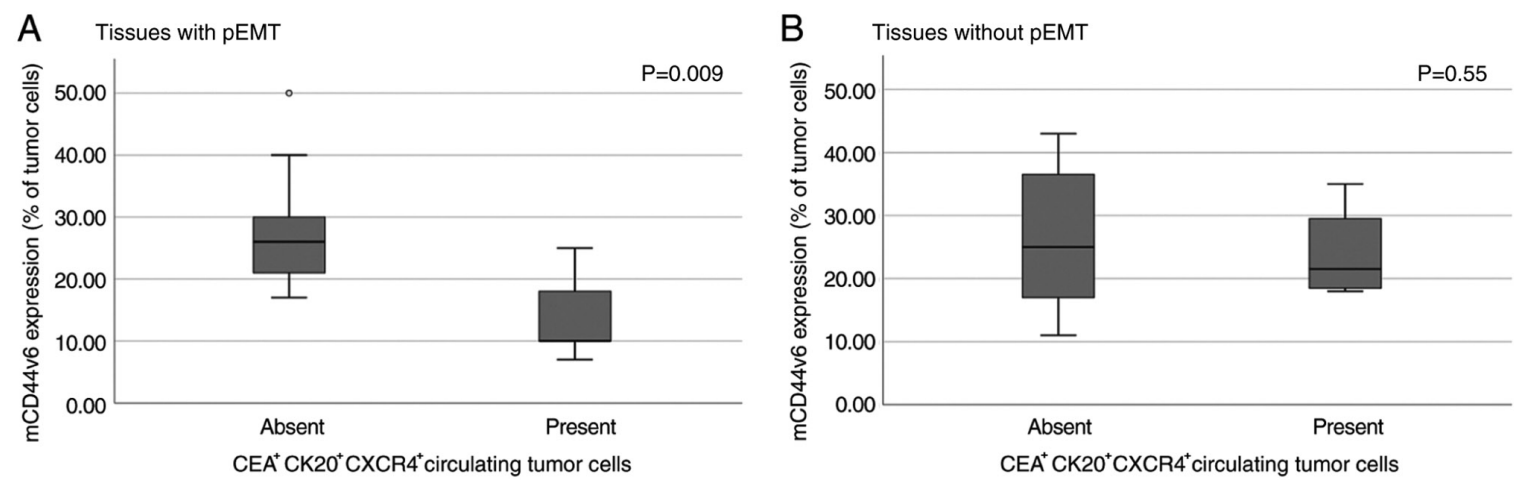

Figure 6. Implication of pEMT in the association between mCD44v6 and CTCs. Association between mCD44v6 expression and circulating tumor cells in the (A) presence and (B) absence of pEMT in primary colorectal cancer tissues. mCD44v6, membranous CD44 variant 6; pEMT, partial epithelial-to-mesenchymal transition.

analysis of metastasis-free survival in relation to mCD44v6 expression (negative vs. positive) is shown in Fig. 7. Patients with negative $\mathrm{mCD} 44 \mathrm{v} 6$ expression were found to exhibit worse prognosis $(\mathrm{P}=0.037)$.
Cells on proliferation (S phase) in negative vs. positive $m C D 44 v 6 C R C$ tissues. Among all primary cancer tissues, the mean percentage of cells on S phase was $24.61 \%$ (17.28). Upon revealing that tissues with negative $(<18.44 \%)$ mCD44v6 
Table II. Simple model analysis of composite endpoint (metastasis or death).

\begin{tabular}{lcc}
\hline Variable & HR & 95\% CI \\
\hline Age, years & 1.021 & $0.924-1.129$ \\
Sex (male vs. female) & 0.570 & $0.142-2.283$ \\
Lymph node metastasis (III vs. 0-II) & 1.306 & $0.312-5.466$ \\
Histological grade & & 0.427 \\
1 & Reference & Reference \\
2 & 1.203 & $0.145-10.005$ \\
3 & 1.675 & $0.104-26.866$ \\
Tumor diameter, cm & 1.307 & $0.979-1.745$ \\
Left vs. right-sided & 3.317 & $0.408-26.995$ \\
CK20 ${ }^{+}$-CEA ${ }^{+}$-CXCR4 ${ }^{+}$CTCs (positive vs. negative) & 1.796 & $0.428-7.534$ \\
pEMT vs. non-pEMT & 1.020 & 0.715 \\
Cells in S phase, $\%$ & 1.027 & $0.244-4.269$ \\
mCD44v6 expression & 0.915 & 0.262 \\
MTA1 expression & 1.005 & 0.423 \\
\hline
\end{tabular}

${ }^{\text {a }}<$ 0.05. HR, hazard ratio; CI, confidence interval; CTCs, circulating tumor cells; pEMT, partial epithelilal-to-mesenchymal transition; mCD44v6, membranous CD44 variant 6; MTA1, metastasis-associated protein 1.

Table III. Multivariable model analysis of composite endpoint (metastasis or death).

\begin{tabular}{lccc}
\hline Variable & HR & $95 \%$ CI & P-value \\
\hline Tumor diameter, cm & 1.363 & $0.994-1.869$ & 0.054 \\
mCD44v6 expression & 0.882 & $0.796-0.978$ & $0.017^{\text {a }}$ \\
\hline
\end{tabular}

${ }^{\mathrm{a}} \mathrm{P}<0.05$. HR, hazard ratio; $\mathrm{CI}$, confidence interval; mCD44v6, membranous CD44 variant 6 .

expression had worse disease-free survival, we further examined the proliferative status of these tissues. In tissues negative for $\mathrm{mCD} 44 \mathrm{v} 6$ expression, the percentage of cells on $\mathrm{S}$ phase was $36.61 \%$ (21.57), median: $36 \%$ whereas in positive ones the respective percentage was $20.61 \%$ (14.01), median: $18.1 \%$. This difference did not reach to a statistically significant level $(\mathrm{P}=0.06)$.

\section{Discussion}

The present study managed to reveal information about the tumor-stroma reciprocal influence during cancer development and metastasis by obtaining 'a snapshot' of mCD44v6 expression from normal epithelium, to benign polyps and cancer lesions with or without potential of disease spreading. In our study we focused on mCD44v6 expression, since CD44v6 contains a cell surface receptor for hyaluronic acid which is a major component of the extracellular matrix $(11,22)$. Advances in the past years have shown that, although hyaluronic acid is a simple linear polymer of $\mathrm{N}$-acetylglucosamine and D-glucuronic acid, it can create a viscous gel by binding water and sequester growth factors and cytokines in the tumor microenvironment (22). Our data showed that mCD44v6 expression increases in CRC, but it is its eventual down-regulation that is associated with CTCs and worse prognosis.

Initially, we demonstrated an increase in the population of cells expressing mCD44v6 in CRC tissues compared to normal tissues or benign polyps, although in the past, opposing findings were also reported. Specifically, Wang et al found that the predominant expression of mCD44v6 occurred in adenomas (23). However, in another study while the positive rates for CD44v6 ranged from 72 to $100 \%$ for tubular, tubulovillous and villous adenomas, hyperplastic polyps were negative (24). Since the majority of the polyps in our study were benign hyperplastic, our findings are in line with the literature. Nonetheless, it should be noted that in several studies increased CD44 expression is considered to be an early event in the adenoma-carcinoma sequence (23-25).

Regarding the clinical significance of CD44v6 in CRC metastasis, studies are contradictory although consistently agreeing on its significant implication. Some studies support CD44v6 upregulation as a poor prognostic indicator $(26,27)$ while others, including the present one, suggest its downregulation as a poor prognostic factor $(23,28,29)$. In an attempt to accumulate literature findings, a meta-analysis showed that CD44v6 overexpression was related to worse prognosis (30). However, a crucial issue that deserves consideration as a potential reason for these contradictory results is that membranous and cytoplasmic CD44v6 expression are not distinguished in many of the published studies. Indeed, when focusing on studies based on mCD44v6 expression, the results are in accordance with the herein presented $(23,29)$. In a systematic assessment of the prognostic impact of mCD44v6 protein, loss of mCD44v6 was associated with lymph node metastasis and adverse prognosis with the reported mean mCD44v6 expression in the primary lesions $(21.8 \%)$ to be very similar to the percentage of $\mathrm{mCD} 44 \mathrm{v} 6$ detected in our patient's cohort, with the reduction in mCD44v6 expression occuring from the 


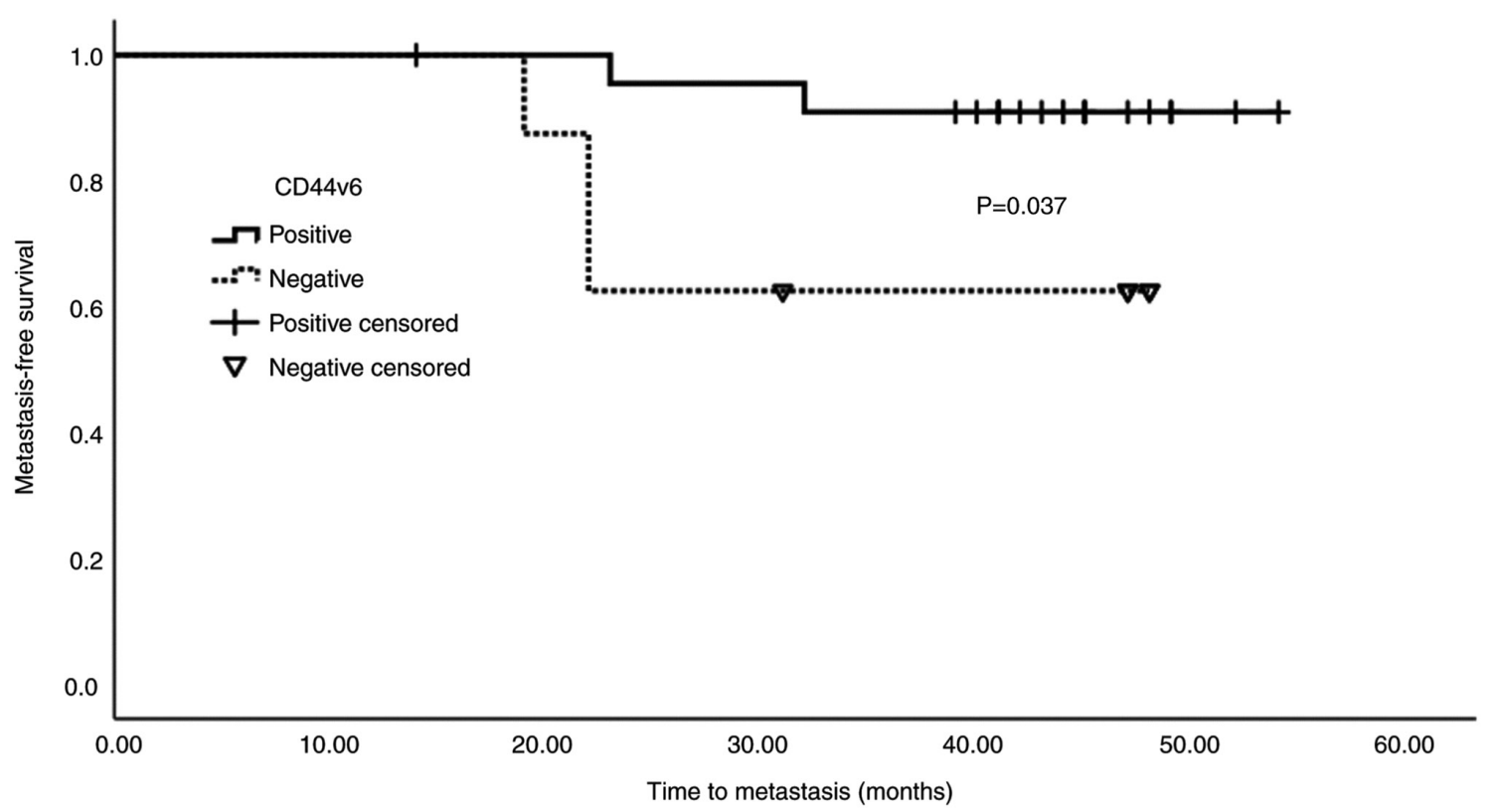

Figure 7. Kaplan-Meier survival curve. Survival analysis demonstrated that downregulated mCD44v6 expression was associated with poor prognosis. The mean value of mCD44v6 expression in patients with colorectal cancer with triple positive circulating tumor cells $\left(\mathrm{CK}_{20} 0^{+} \mathrm{CEA}^{+} \mathrm{CXCR} 4^{+}\right)$was used to characterize patients as positive $(\geq 18.44 \% ; \mathrm{n}=23)$ or negative $(<18.44 \% ; \mathrm{n}=8)$. $\mathrm{mCD} 44 \mathrm{v} 6$, membranous CD44 variant 6 .

center of the tumor to its invasive front (29). Due to the nature of the methodology used for tissue analysis, a topographical distinction of mCD44v6 expression between the center and the tumor margin was not possible in the present study. Finally, Coppola et al observed a gradual loss of mCD44v6 during CRC progression (28). Our data support the association of reduced mCD44V6 expression with worse prognosis, as cancer lesions with low mCD44v6 expression tended to present enhanced proliferative characteristics based on the percentage of cells on the $\mathrm{S}$ phase and their association with the presence of CTCs. The latter association was more significant in the presence of pEMT, which is line with the notion that cells on pEMT have distinct advantages in terms of metastasis. Their mesenchymal phenotype allows their entrance into the bloodstream, while their epithelial phenotype allows them to move in clusters enhancing tumor-seeding properties $(6,7)$. For the detection of CTCs in our cohort of CRC patients all measurements were taken in order to avoid any exogenous epithelial contamination and the expression of CXCR4 was explored in order to further identify CTCs with increased metastatic potential (19). Several studies further support that CD44v6 itself may induce the EMT phenotype $(8,31)$, leaning towards the hypothesis that CD44v6 upregulation is an early event occurring before EMT in the adenoma-carcinoma sequence of CRC (23-25,28).

Regarding the role of tumor sidedness on $\mathrm{mCD} 44 \mathrm{v} 6$ expression, it has been previously reported that CD44v6 presents reduced expression in distal (left) colon cancers (32). Moreover, left-sided CRC is associated with worse prognosis, mainly based on a study of 91,416 stage I-III CRC patients (33). The data presented here show that lower expression of mCD44v6 was associated with both left-sided tumors as well as worse prognosis, further supporting the observations reported in these studies.

In contrast to the membranous role of CD44v6, MTA1 protein exhibits both nuclear and cytoplasmic localization during cancer progression $(14,34)$ which could not be distinguished in the present study and is a drawback that may explain why in our cohort of patients MTA1 was not a prognostic factor as it has been previously demonstrated for other types of cancer $(35,36)$. Still, the herein presented results showed an increase in MTA1 levels during the transition from normal/benign tissue to CRC which is line with previously reported data (35,37). Finally, the correlation of MTA1 and mCD44v6 expression profiles may be explained through the results of a previous study which showed that CD44 up-regulates MTA1 expression via HER2 (38).

A major limitation of our study that deserves consideration is the small patient database, which may have limited the generalizability of our results. However, we believe that the remarkable advantage of our study, when compared to previous ones, is that it managed to indicate that both increased and decreased CD44v6 expression are associated with CRC progression and furthermore to propose a justification for the contradictory findings in the literature regarding the clinical significance of this molecule.

In conclusion, our results revealed that both MTA1 and $\mathrm{mCD} 44 \mathrm{v} 6$ expressions were increased in malignant colorectal tumors when compared to normal colorectal tissue or benign hyperplastic polyps, with their expression highly correlating. Furthermore, reduced mCD44v6 expression was linked to the presence of CTCs, as well as a worse metastasis-free survival. The distinctive analysis of membranous, as opposed to cytoplasmic CD44v6 may explain previous contradictive results. Considering that in the presence of pEMT, where cells lose the cell to cell adhesion, this association was even stronger, it is proposed that reduced $\mathrm{mCD} 44 \mathrm{v} 6$ expression (which adheres to the hyaluronic acid) is associated with pEMT and may lead to a relaxed connection of cancer cells with the extracellular matrix, conferring migratory freedom and increasing their mobility and metastatic potential. Overall, the observed 
overexpression of mCD44v6 in CRC and the eventual downregulation of $\mathrm{mCD} 44 \mathrm{v} 6$ in the presence of CTCs can represent a point of encouragement for the assessment of relevant therapeutic strategies in the era of precision medicine.

\section{Acknowledgements}

The abstract was presented at the Young Oncologist Award Competition in 2020 (Zilina, Slovakia).

\section{Funding}

The present study was supported by the VISION project (strategies to strengthen scientific excellence and innovation capacity for early diagnosis of gastrointestinal cancers), which received funding from the European Union's Horizon 2020 research and innovation programme (grant no. 857381).

\section{Availability of data and materials}

The dataset used and/or analyzed during the current study are available from the corresponding author on reasonable request.

\section{Authors' contributions}

AK, VGG, AD and MK conceived the present study. AK, VGG and AD performed the experiments. KP provided polyp bioptic material. EK performed histological confirmation and immunohistochemical analysis. EK, KP, AD, AK and VGG analyzed the data and drafted the initial manuscript. AD, KP, EK and MK critically revised the manuscript for important intellectual content. AK and VGG confirmed the authenticity of all the raw data. AK and MK supervised the project. All authors have read and approved the final manuscript.

\section{Ethics approval and consent to participate}

The present study was approved by Hippokratio Hospital's Ethics Committee (approval no. 18601/2019) and patients provided written informed consent prior to the study start.

\section{Patient consent for publication}

Not applicable.

\section{Competing interests}

The authors declare that they have no competing interests.

\section{References}

1. Rawla P, Sunkara T and Barsouk A: Epidemiology of colorectal cancer: Incidence, mortality, survival, and risk factors. Prz Gastroenterol 14: 89-103, 2019.

2. De Palma FDE, D'argenio V, Pol J, Kroemer G, Maiuri MC and Salvatore F: The molecular hallmarks of the serrated pathway in colorectal cancer. Cancers (Basel) 11: 1017, 2019.

3. Okabe H, Mima K, Saito S, Hayashi H, Imai K, Nitta H, Hashimoto D, Chikamoto A, Ishiko T, Beppu T and Baba H: Epithelial-mesenchymal transition in gastroenterological cancer. J Cancer Metastasis Treat 1: 183-189, 2015.
4. Loh CY, Chai JY, Tang TF, Wong WF, Sethi G, Shanmugam MK, Chong PP and Looi CY: The E-Cadherin and N-Cadherin Switch in Epithelial-to-Mesenchymal transition: Signaling, therapeutic implications, and challenges. Cells 8: 1118, 2019.

5. Nieto MA, Huang RY, Jackson RA and Thiery JP: EMT: 2016. Cell 166: 21-45, 2016.

6. Jolly MK, Boareto M, Huang B, Jia D, Lu M, Ben-Jacob E, Onuchic $\mathrm{JN}$ and Levine $\mathrm{H}$ : Implications of the hybrid epithelial/mesenchymal phenotype in metastasis. Front Oncol 5: 155,2015

7. Aiello NM and Kang Y: Context-dependent EMT programs in cancer metastasis. J Exp Med 216: 1016-1026, 2019.

8. Saito S, Okabe H, Watanabe M, Ishimoto T, Iwatsuki M, Baba Y, Tanaka Y, Kurashige J, Miyamoto Y and Baba H: CD44v6 expression is related to mesenchymal phenotype and poor prognosis in patients with colorectal cancer. Oncol Rep 29: $1570-1578,2013$

9. Tuncay Cagatay S, Cimen I, Savas B and Banerjee S: MTA-1 expression is associated with metastasis and epithelial to mesenchymal transition in colorectal cancer cells. Tumor Biol 34: 1189-1204, 2013.

10. Wang Z, Zhao K, Hackert T and Zöller M: CD44/CD44v6 a reliable companion in cancer-initiating cell maintenance and tumor progression. Front Cell Dev Biol 6: 97, 2018.

11. Chen C, Zhao S, Karnad A and Freeman JW: The biology and role of CD44 in cancer progression: Therapeutic implications. J Hematol Oncol 11: 64, 2018.

12. McAtee CO, Barycki JJ and Simpson MA: Emerging roles for hyaluronidase in cancer metastasis and therapy. Adv Cancer Res 123: 1-34, 2014.

13. Misra S, Hascall VC, Markwald RR and Ghatak S: Interactions between hyaluronan and its receptors (CD44, RHAMM) regulate the activities of inflammation and cancer. Front Immunol 6: 201, 2015.

14. Liu J, Xu D, Wang H, Zhang Y, Chang Y, Zhang J, Wang J, Li C, Liu H, Zhao M, et al: The subcellular distribution and function of MTA1 in cancer differentiation. Oncotarget 5: 5153-5164, 2014.

15. Lozar T, Gersak K, Cemazar M, Kuhar CG and Jesenko T: The biology and clinical potential of circulating tumor cells. Radiol Oncol 53: 131-147, 2019.

16. Wang Y, Zhou Y and Hu Z: The functions of circulating tumor cells in early diagnosis and surveillance during cancer advancement. J Transl Intern Med 5: 135-138, 2017.

17. Welinder C, Jansson B, Lindell G and Wenner J: Cytokeratin 20 improves the detection of circulating tumor cells in patients with colorectal cancer. Cancer Lett 358: 43-46, 2015.

18. Lagoudianakis EE, Kataki A, Manouras A, Memos N, Papadima A, Derventzi A, Zografos G, Papadopoulos S, Katergiannakis V and Konstadoulakis MM: Detection of epithelial cells by RT-PCR targeting CEA, CK20, and TEM-8 in colorectal carcinoma patients using OncoQuick density gradient centrifugation system. J Surg Res 155: 183-190, 2007.

19. Miller RJ, Banisadr G and Bhattacharyya BJ: CXCR4 signaling in the regulation of stem cell migration and development. J Neuroimmunol 198: 31-38, 2008.

20. Riihimäki M, Hemminki A, Sundquist J and Hemminki K: Patterns of metastasis in colon and rectal cancer. Sci Rep 6: 29765, 2016.

21. Liepelt A and Tacke F: Stromal cell-derived factor-1 (SDF-1) as a target in liver diseases. Am J Physiol Gastrointest Liver Physiol 311: G203-G209, 2016.

22. Werb $\mathrm{Z}$ and Lu P: The role of Stroma in tumor development. Cancer J 21: 250-253, 2015.

23. Wang L, Liu Q, Lin D and Lai M: CD44v6 down-regulation is an independent prognostic factor for poor outcome of colorectal carcinoma. Int J Clin Exp Pathol 8: 14283-14293, 2015.

24. Afify A, Durbin-Johnson B, Virdi A and Jess H: The expression of CD44v6 in colon: From normal to malignant. Ann Diagn Pathol 20: 19-23, 2016.

25. Kim H, Yang XL, Rosada C, Hamilton SR and August JT: CD44 expression in colorectal adenomas is an early event occurring prior to K-ras and p53 gene mutation. Arch Biochem Biophys 310: 504-507, 1994.

26. Todaro M, Gaggianesi M, Catalano V, Benfante A, Iovino F, Biffoni M, Apuzzo T, Sperduti I, Volpe S, Cocorullo G, et al: $\mathrm{CD} 44 \mathrm{v} 6$ is a marker of constitutive and reprogrammed cancer stem cells driving colon cancer metastasis. Cell Stem Cell 14: 342-356, 2014. 
27. Zhao LH, Lin QL, Wei J, Huai YL, Wang KJ and Yan HY: CD44v6 expression in patients with stage II or stage III sporadic colorectal cancer is superior to CD44 expression for predicting progression. Int J Clin Exp Pathol 8: 692-701, 2015.

28. Coppola D, Hyacinthe M, Fu L, Cantor AB, Karl R, Marcet J, Cooper DL, Nicosia SV and Cooper HS: CD44V6 expression in human colorectal carcinoma. Hum Pathol 29: 627-635, 1998.

29. Zlobec I, Günthert U, Tornillo L, Iezzi G, Baumhoer D, Terracciano L and Lugli A: Systematic assessment of the prognostic impact of membranous CD44v6 protein expression in colorectal cancer. Histopathology 55: 564-575, 2009.

30. Wang JL, Su WY, Lin YW, Xiong H, Chen YX, Xu J and Fang JY: CD44v6 overexpression related to metastasis and poor prognosis of colorectal cancer: A meta-analysis. Oncotarget 8: 12866-12876, 2017.

31. Okabe H, Ishimoto T, Mima K, Nakagawa S, Hayashi $H$, Kuroki H, Imai K, Nitta H, Saito S, Hashimoto D, et al: CD44s signals the acquisition of the mesenchymal phenotype required for anchorage-independent cell survival in hepatocellular carcinoma. Br J Cancer 110: 958-966, 2014.

32. Minoo P, Zlobec I, Peterson M, Terracciano L and Lugli A: Characterization of rectal, proximal and distal colon cancers based on clinicopathological, molecular and protein profiles. Int J Oncol 37: 707-718, 2010.

33. Warschkow R, Sulz MC, Marti L, Tarantino I, Schmied BM, Cerny $\mathrm{T}$ and Güller U: Better survival in right-sided versus left-sided stage I-III colon cancer patients. BMC Cancer 16: 554, 2016.
34. Aramaki Y, Ogawa K, Toh Y, Ito T, Akimitsu N, Hamamoto H, Sekimizu K, Matsusue K, Kono A, Iguchi $\mathrm{H}$ and Takiguchi S: Direct interaction between metastasis-associated protein 1 and endophilin 3. FEBS Lett 579: 3731-3736, 2005.

35. Cao G, Chen B and Xiong M: Role of metastasis-associated protein 1 in prognosis of patients with digestive tract cancers: A meta-analysis. PLoS One 12: e0176431, 2017.

36. Karamagkiolas S, Giotakis I, Kyrodimos E, Giotakis EI, Kataki A, Karagianni F and Lazaris AM: Expression of vimentin (VIM) and metastasis-associated 1 (MTA1) protein in laryngeal squamous cell carcinoma are associated with prognostic outcome of patients. Am J Otolaryngol 40: 487-493, 2019.

37. Chen XL, Wu YJ, Lv Z, Zhao HM and Han BJ: Significance of expression of MTA1 and RECK in carcinogenesis of colonic polyps. World Chinese J Dig 24: 4514-4518, 2016.

38. Bao W, Fu HJ, Xie QS, Wang L, Zhang R, Guo ZY, Zhao J, Meng YL, Ren XL, Wang T, et al: HER2 interacts with CD44 to up-regulate CXCR4 via epigenetic silencing of microRNA-139 in gastric cancer cells. Gastroenterology 141: 2076-2087.e6, 2011. International (CC BY-NC-ND 4.0) License. 